\title{
FACTORES ALIMENTARIOS Y DIETÉTICOS ASOCIADOS A LA OBESIDAD INFANTIL: RECOMENDACIONES PARA SU PREVENCIÓN ANTES DE LOS DOS AÑOS DE VIDA
}

\author{
FOOD AND DIET FACTORS ASSOCIATED TO CHILD OBESITY: \\ RECOMMENDATIONS FOR PREVENTING IT IN CHILDREN UNDER \\ TWO YEARS OF AGE
}

José M. Saavedra ${ }^{1,2, a}$, Anne M. Dattilo,

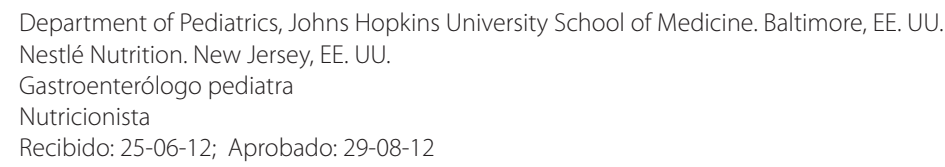

\section{RESUMEN}

Son varios los factores identificados que llevan a la obesidad, cuyo origen se da desde etapas muy tempranas de la vida. Aunque existen contribuyentes genéticos y disparidades socioeconómicas y raciales en la frecuencia de sobrepeso y obesidad, el problema es universal. El estado ponderal de los niños se ha asociado con múltiples factores dietéticos específicos y estos, a su vez, están asociados con específicas "prácticas de alimentación por parte de los padres" (PAPP). Los padres o cuidadores determinan patrones que se establecen muy temprano en la vida, y el peso antes de los dos años de vida predice ya el sobrepeso u obesidad futura. La falta de lactancia materna y la baja calidad de los alimentos, los patrones de consumo y las preferencias por alimentos en los primeros dos años de vida, son altamente dependientes de las PAPP y, por tanto, son también modificables. En este artículo vamos a examinar los factores dietéticos y las prácticas relacionadas con la dieta que han sido asociadas con la obesidad infantil, y que son potencialmente modificables, de forma que sirvan como guía potencial para metas de intervención, en la prevención de la obesidad infantil.

Palabras clave: Obesidad; Sobrepeso; Niño; Infante; Alimentación; Nutrición infantil (fuente: DeCS BIREME).

\begin{abstract}
Several factors leading to obesity have been identified, which start at very early stages of life. Although there are genetic contributors and socioeconomic and racial disparities in the prevalence of overweight and obesity, the problem is universal. Children's weight has been associated to multiple specific diet factors and these, in turn, are associated to specific "parent feeding practices" (PAPP, Spanish abbreviation). Parents or caretakers determine patterns that are set at an early stage in life, and the weight before the first two years of age already predicts future overweight and obesity. Absence of breastfeeding and low food quality, consumption patterns and food preferences in the first two years of life are highly associated to PAPP and can, therefore, be modified. In this article, we will examine the diet factors and the diet-related practices that have been associated to child obesity, and are potentially modifiable, so that they can be used as a potential guide for intervention goals in the prevention of child obesity.
\end{abstract}

Key words: Obesity; Overweight; Child; Child, Preschool; Feeding; Infant nutrition (source: MeSH NLM).

Citar como: Saavedra JM, Dattilo AM. Factores alimentarios y dietéticos asociados a la obesidad infantil: recomendaciones para su prevención antes de los dos años de vida. Rev Peru Med Exp Salud Publica. 2012;29(3):379-85. 


\section{MAGNITUD, DISTRIBUCIÓN Y ENFOQUE DEL PROBLEMA}

La prevalencia del sobrepeso y la obesidad en los niños ha aumentado dramáticamente en los últimos decenios. Aumentos en peso, talla, y en el índice de masa corporal (IMC), se observan en los niños y niñas de todo el mundo. Estimados de 34 países miembros de la Organización para la Cooperación Económica y Desarrollo (OCE) muestran que $21 \%$ de las niñas y el $23 \%$ de los niños tienen sobrepeso ${ }^{(1)}$. Estas cifras han alcanzado proporciones epidémicas en varios países, con estimados de sobrepeso en el $45 \%$ de los varones en Grecia y casi el $30 \%$ de los niños en Chile, Nueva Zelanda, México, Italia y Estados Unidos (EE. UU.). Un estudio reciente en EE. UU. identifica que en una muestra grande de niños nacidos en el 2001, a los nueve meses de edad, aproximadamente un tercio ya tenían sobrepeso (> 85 percentil de IMC para edad) u obesidad (> 95 percentil) ${ }^{(2)}$

La adiposidad infantil o el IMC incrementado, tan temprano como a las dos semanas de edad (3), y en los primeros 24 meses de edad ${ }^{(4,5)}$, están asociados con un aumento significativo en el riesgo de sobrepeso durante la edad preescolar. Los niños que son obesos a los nueve o veinticuatro meses, tienen tres veces más probabilidad de mantener este sobrepeso a la edad de cuatro años, en comparación con niños no obesos durante los dos primeros años de vida. En contraste, niños con un peso normal a los nueve meses de vida, permanecen con un peso normal hasta los dos años, y tienen mayor probabilidad de mantenerse con peso normal $(84,8 \%)$ que de desarrollar sobrepeso $(8,9 \%)$, u obesidad $(6,3 \%)$ a los cuatro años de vida ${ }^{(4)}$. El estado ponderal del lactante predice el peso del niño más tarde, y los cambios en el IMC durante años preescolares se correlacionan fuertemente con el sobrepeso en adultos. Por tanto, el desarrollo ponderal en la infancia es un momento crítico y de gran potencial para la prevención.

El estado socioeconómico, a menudo, se encuentra asociado con la prevalencia de obesidad en países desarrollados, y tendencia a mayor obesidad en las clases socioeconómicas más bajas. Sin embargo, también se han observado inconsistencias en la dirección de esta asociación, dependiendo de raza, etnicidad, sexo, y desarrollo económico ${ }^{(6)}$. En un estudio en el Perú, entre 2007-2010, con una muestra de 3669 niños menores de cinco años, se encontró una prevalencia nacional de sobrepeso y obesidad del 6,9\%. Los niños en Lima Metropolitana tuvieron la mayor prevalencia de exceso de peso $(10,1 \%)$ en comparación con la prevalencia de solo $2,6 \%$ en niños en la selva ${ }^{(7)}$.
La relación entre estado socioeconómico en los niños está menos estudiada que en los adultos; sin embargo, al menos tres estudios del Brasil muestran una mayor prevalencia de obesidad en niños de familias con mayores ingresos ${ }^{(8-10)}$, llegando hasta el $34,4 \%$ en niños y niñas entre 2-6 años de edad, en una muestra de niños con escolaridad privada en Sao Paulo (10).

En una encuesta nacional de niños en edad escolar, también del Brasil en 2002-2003, los niños de los quintiles más altos de la distribución de ingresos tuvieron aproximadamente tres veces más probabilidades de tener sobrepeso u obesidad $(19,2 \%)$ que en los quintiles más bajos $(6,2 \%)$. Para las niñas, los quintiles más altos de ingresos incrementaron en dos veces la probabilidad de tener sobrepeso u obesidad $(14,9 \%)$ que los de bajos quintiles (8,5\%). En niños y niñas 10 a 14 años de edad el incremento de sobrepeso y la obesidad correlacionaron bien con el incremento de ingreso familiar ${ }^{(11)}$.

Los datos disponibles son más limitados en cuanto a grupos raciales y étnicos en diferentes países. Sin embargo, datos de EE. UU. muestran que algunos subgrupos de niños experimentan una mayor prevalencia de sobrepeso y obesidad que otros. Por ejemplo, en 2002, niños de origen hispanoamericano mostraron la mayor tasa de sobrepeso y obesidad en comparación con niños de otros orígenes, a los nueve meses y dos años de vida ${ }^{(2)}$. Estimados de prevalencia más recientes en los EE. UU. confirman que los niños hispanos y mexicanoestadounidenses tienen tasas de sobrepeso y obesidad desproporcionadamente altas en comparación con otros grupos. En 2007-2008, el 40\% de niños mexicanoestadounidenses en edad escolar tenían sobrepeso, en comparación con un promedio nacional de $32 \%$ en todos los niños dentro de este grupo etario. De mayor preocupación es la prevalencia de obesidad en lactantes del mismo origen, que supera el $12 \%{ }^{(12)}$.

Las diferencias genéticas, raciales, étnicas y geográficas mencionadas anteriormente constituyen factores de riesgo de obesidad no modificables. Hay también factores asociados al estado ponderal y metabólico de la madre que influyen el crecimiento y programación metabólica in-útero; los cuales podrían ser parcialmente modificables.

Finalmente, hay claros factores ambientales, comerciales y culturales que influencian la dieta, la actividad y el ejercicio. Si bien estos son de gran importancia, tienen su mayor efecto en niños mayores y adultos en un momento para el cual ya se han establecido en gran medida los patrones de preferencias alimentarias y de consumo asociadas con la obesidad pediátrica, y estamos viendo los efectos de estos patrones en el 
incremento de los índices de obesidad en lactantes y en preescolares. Si hemos de prevenir el progreso de la epidemia, es crítico examinar nuestras posibilidades de prevención en la vida temprana.

En ese artículo resumimos los factores dietéticos potencialmente modificables en la infancia, que se han asociado específicamente con un ritmo acelerado de ganancia de peso para edad, peso para talla, IMC y otras medidas de adiposidad en la infancia ${ }^{(13)}$. En particular, examinamos los factores dietéticos y las prácticas de alimentación por parte de los padres (PAPP), que han sido identificados como contribuyentes a la obesidad infantil.

\section{FACTORES ALIMENTARIOS Y DIETÉTICOS ASOCIADOS AL SOBREPESO Y LA OBESIDAD INFANTIL}

\section{LACTANCIA MATERNA}

Son varios los estudios de observación que concluyen que los lactantes alimentados con leche materna tienen menor riesgo de sobrepeso, en comparación con los lactantes alimentados con fórmulas infantiles. Aunque algunos resultados tempranos no mostraron consistencia en sus resultados, estudios más recientes concluyen con mayor certidumbre que la lactancia materna exclusiva, y la mayor duración de exclusividad, están inversamente asociadas con la tasa de ganancia de peso, peso corporal, adiposidad o riesgo de sobrepeso y obesidad en el niño y los niños de edad preescolar ${ }^{(14)}$. A pesar de los beneficios salubres de la lactancia materna, las tasas de lactancia materna exclusiva continúan desalentadoramente bajas, llegando globalmente a solo $34,6 \%$ de los recién nacidos a los seis meses de edad ${ }^{(15)}$.

Los mecanismos por los cuales la lactancia materna puede disminuir el riesgo de sobrepeso o obesidad son multifactoriales y no claramente dilucidados, pero es aparente que la composición de la leche materna determina la programación de procesos metabólicos en el lactante, e incluso modula el número y el tamaño de los adipocitos. El incremento de la ingesta de energía, proteínas, o ambos, asociados con la alimentación de fórmula infantil se ha propuesto como factor contribuyente al desarrollo de obesidad ${ }^{(16)}$. Es igualmente posible que los lactantes amamantados desarrollen un estilo de alimentación que permite una mayor autorregulación de ingesta en comparación con la alimentación por biberón. Por ejemplo, en un estudio reciente, bebés alimentados exclusivamente con leche materna por biberón ganaron significativamente más peso que bebés alimentados directamente de la mama ${ }^{(17)}$. El mismo acto de alimentar con biberón puede afectar la percepción y la interpretación por parte de la madre de las señales de hambre y saciedad del lactante. En el niño amamantado, estas relaciones están muy bien reguladas y no interfieren significativamente con los mecanismos de oferta y demanda del amamantamiento.

La lactancia materna también se ha asociado con otras prácticas alimentarias deseables, que están relacionadas con un menor riesgo de sobrepeso, tal como la introducción más tardía de alimentos complementarios (después de los cinco o seis meses) y consumo menos frecuente de alimentos y bebidas azucaradas, y de alimentos altos en grasas antes del primer año de vida ${ }^{(18)}$. Además, la lactancia materna proporciona al lactante una amplia gama de sabores derivados de los alimentos ingeridos por la madre, que a su vez pueden influir en la aceptación posterior y la preferencia de estos sabores en los alimentos de destete. Estas primeras experiencias con compuestos de sabor en la leche materna parecen modificar la aceptación posterior de alimentos por parte del lactante, potencialmente modulando sus preferencias alimentarias a largo plazo ${ }^{(19)}$. Esto podría también explicar el hallazgo de que los bebés amamantados tienden a ser con menos frecuencia "comedores quisquillosos" y a estar más dispuestos a probar nuevos alimentos.

\section{DIETA: CALIDAD Y CANTIDAD}

Los datos más recientes y más completos sobre la calidad de la dieta de niños de 0 a 4 años de vida, se encuentran en el estudio Feeding Infants and Toddlers Study (FITS); la cual es una encuesta alimentaria transversal a más de 3000 niños, a nivel nacional en los EE. UU., llevada a cabo $2008^{(20)}$. Los resultados indican que la ingesta de energía promedio es mayor que los requerimientos estimados, en todas las edades estudiadas. $Y$ este exceso empieza temprano. Los lactantes menores de seis meses de edad mostraron una ingesta energética $14 \%$ mayor que los requerimientos estimados para esa población (83 kcal/día de exceso).

El consumo de alimentos azucarados (postres) y bebidas tiene una correlación positiva con la incidencia de sobrepeso y obesidad en preescolares y niños mayores (21). Aquí también, el problema empieza temprano. En la encuesta FITS, se encontró que en un día tomado al azar el $45 \%$ de los lactantes entre 9-12 meses de edad, y dos tercios de lactantes a los 18 meses de vida consumían postres y bebidas azucaradas ${ }^{(22)}$. Este nivel de consumo se mantiene constante y constituye el $12 \%$ de la ingesta energética total en los primeros tres años de vida (Figura 1). 


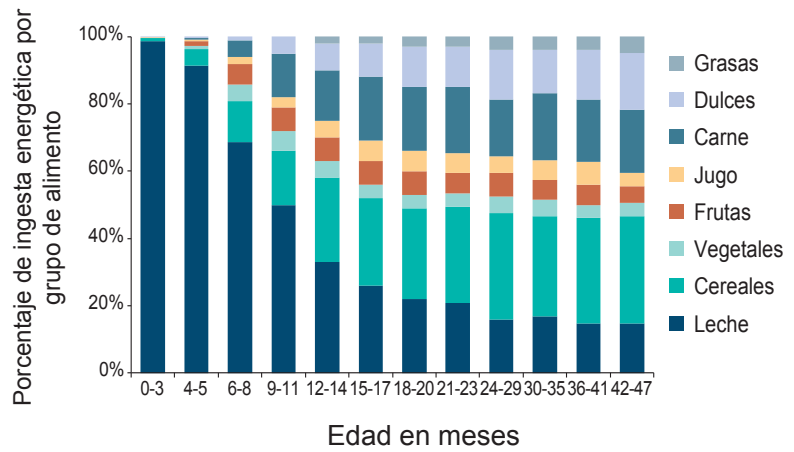

Figura 1. Ingesta energética relativa por grupo de alimentos en los primeros cuatro años de vida.

Dulces: incluye galletas, golosinas, tortas.

Fuente: elaboración propia a partir de los datos de "Siega-Riz et al. Food consumption patterns of infants and toddlers: where are we now? J Am Diet Assoc 2010;110:S38-S51".

El estudio FITS muestra que los patrones dietarios asociados con el sobrepeso surgen muy temprano y se mantienen. En otro día escogido al azar, se encontró que aproximadamente el $37 \%$ de niños de 6-9 meses de edad y el $28 \%$ de los niños de 9-15 meses de edad no consumieron ni una sola porción de verduras ${ }^{(22)}$. En los niños que sí consumen verduras, la cantidad disminuyó de 84 g/día en lactantes de más de seis meses, a solo $64 \mathrm{~g} /$ día en niños de 12 a 24 meses, y permaneció en este nivel de ingesta hasta los cuatro años de edad. A partir de los 18 meses de edad y hasta los cuatro años, las frutas y verduras representaron solo el 7 y $5 \%$ del aporte energético total respectivamente. Después del año de vida, la papa blanca remplaza a la mayor parte de vegetales consumidos. Más aun, para los dos años de vida, 2/3 de la papa consumida por niños norteamericanos es en forma de papas fritas. Un hallazgo muy importante de este estudio es el hecho de que a pesar del incremento en ingesta energética total y del incremento en la variedad de alimentos entre los 18 meses y los cuatro años de vida, la "contribución energética relativa" de cada grupo de alimentos (lácteos, cereales, carnes, frutas, verduras, etc.) se mantiene sorprendentemente estable (Figura 1); y esta distribución es ya representativa de la distribución de ingesta de grupos alimentarios en adultos en EE. UU. Esto indica que los patrones de preferencia por grupo alimentario, se fijan muy tempranamente en la vida.

La ingesta energética relativa cambia rápidamente con la introducción de alimentos sólidos entre los 4 y los 18 meses de vida, volviéndose notablemente constante, hasta los cuatro años de edad. El patrón a los cuatro años es similar al de adultos en Norte América. Datos del estudio FITS - EE. UU. (Feeding Infants and Toddler Study, 2008) ${ }^{(23)}$.
En resumen, análisis de estos y otros datos sugiere que los patrones dietéticos obesogénicos se establecen muy tempranamente, en los primeros dos años de vida y la calidad de la dieta empieza a declinar tan pronto el niño adopta la dieta adulta.

\section{APRENDIZAJE DE SABORES Y PREFERENCIAS ALIMENTARIAS}

Los humanos nacen con preferencias innatas al sabor dulce y al salado. La parcialidad al dulce, presente en el neonato, es universalmente mayor en niños, en comparación con adultos, como es evidente en estudios en varios países y culturas ${ }^{(24)}$. Asimismo, los recién nacidos tienen un gusto innato por el sabor salado y una aversión inicial por el sabor amargo y el ácido. Desde una perspectiva evolutiva, tendrían sentido estas preferencias por sabores dulces (como en la leche materna y por la energía disponible en hidratos de carbono y las frutas) y por sabores salados (asociados a fuentes de minerales), así como el rechazo a sabores amargos (presentes en toxinas y venenos. Por tanto, mientras la preferencias de alimentos dulces y salados facilitan la aceptación de ciertos alimentos, usualmente de alta densidad calórica, la aceptación de los alimentos de sabor amargo (como algunas frutas y verduras verdes), que ayudan a disminuir la densidad calórica de la dieta, tienen que ser aprendidos en edad temprana, mediante la oferta de estos alimentos por los padres ${ }^{(24,25)}$.

Independiente de preferencias innatas, el aprendizaje de sabores empieza antes del nacimiento. Ciertos sabores consumidos por la madre están presentes en el líquido amniótico. Y ciertos sabores, consumidos por la madre durante el embarazo, son preferidos poco después del nacimiento (medidos por reacciones de movimiento oral y de succión), en comparación con otros sabores ${ }^{(26)}$. Esta exposición temprana a sabores puede tener efectos duraderos.

La lactancia materna ofrece otro modo de aprendizaje. Se ha demostrado que varios sabores pasan a través de la leche materna (por ejemplo, ajo, anís, menta, vainilla y ciertos quesos). Los recién nacidos detectan los sabores en la leche materna como es evidente por cambios en la frecuencia de movimientos de succión, duración del amamantamiento, e ingesta. Por tanto, esta exposición temprana a ciertos sabores puede proporcionar las primeras lecciones de sabor y aumentar la probabilidad de aceptación de ciertos alimentos.

La introducción de alimentos durante el periodo de ablactancia ofrece una ventana crítica para la aceptación potencial de sabores asociados con alimentos 'saludables', en particular frutas y vegetales, ya que 
todos los alimentos sólidos son 'nuevos' para el lactante, y ya que durante esta etapa, la neofobia a alimentos nuevos es relativamente débil. Tanto la introducción temprana a sabores, como la variedad, facilitan la aceptación de alimentos nuevos. La exposición variada y repetida, ofreciendo alimentos que a menudo son inicialmente rechazados (como vainitas verdes, arvejas, y calabaza) durante seis días consecutivos, incrementa significativamente la aceptación y la ingesta de estos alimentos (27).

\section{PRACTICAS DE ALIMENTACIÓN POR PARTE DE LOS PADRES (PAPP)}

Los padres son parte integral del proceso que ayuda a los niños a aceptar sabores y alimentos. Este proceso influencia significativamente qué, cuánto, y cómo los niños aprenden a comer. Por lo tanto, las PAPP durante la primera infancia tienen el potencial de facilitar o dificultar el desarrollo de patrones apropiados de autorregulación de ingesta energética, de preferencias alimentarias y, eventualmente, de influir la elección independiente de los alimentos y la dieta del niño mayor, hasta la adultez.

En el contexto de sobrepeso y obesidad infantil, las prácticas apropiadas de alimentación por parte de los padres (PAAP) (responsive feeding practices), se refieren a una respuesta apropiada a las señales de hambre y saciedad, de acuerdo con el desarrollo físico y emocional del lactante, que promueven una ingesta autorregulada. Estas prácticas incluyen la prestación por parte de los padres de un entorno emocionalmente propicio, con reciprocidad y cierto grado de predictibilidad en las interacciones entre el niño y los padres. Los entornos en los cuales se le alimenta al lactante, que son consistentes con PAPP apropiadas, incluyen calidez, aceptación, y empatía en las respuestas de los padres. Las PAPP no apropiadas toman la forma de un control excesivo (como indebidamente ofreciendo o presionando al niño a terminar el biberón o el plato de comida), o restringiendo excesivamente ciertos alimentos (prohibiendo completamente algún alimento); o por falta de control o involucramiento, con respuestas desinteresada o indulgencia excesiva por parte de los padres ${ }^{(28)}$. Estos factores han sido revisados en mayor detalle en el artículo por Black M y Creed HM en este Simposio.

Los lactantes y niños de edad temprana tienen una capacidad innata para regular el consumo de energía, sin embargo, ciertas PAPP se han asociado con diferencias individuales en la autorregulación de ingesta energética. Hay evidencia de que ciertas PAPP inadecuadas pueden llevar al desaprendizaje de la regulación de la ingesta con el apetito, y las buenas intenciones de los padres pueden alterar el control innato de la ingesta energética del lactante ${ }^{(29)}$. Cuando la alimentación cesa al momento que muestran señales tempranas de saciedad, los lactantes aprenden que sus señales de comunicación de saciedad están siendo "escuchadas" y que lo padres responden a estas muestras de regulación de ingesta. Sin embargo, incluso padres que perciben cuando sus bebés están satisfechos, hacen que el lactante acabe el biberón que se ha preparado ${ }^{(30)}$. Presionar a un bebé así no solo aumenta su ingesta energética, sino hace que el lactante pierda control de su ciclo de hambre y saciedad, con efectos potenciales a más largo plazo.

El alimento se utiliza a menudo para calmar a un bebé "fastidiado" o "cansado". Se podría decir que el alimento es el somnífero para lactantes más comúnmente utilizado a nivel universal, particularmente en lactantes alimentados con biberón. Algunos padres incorrectamente creen que si un bebé llora o esta "quisquilloso" o "fastidiado", lo más probable es que es que tiene hambre, o que el despertarse de noche es una señal de hambre. Esto lleva a la llamada alimentación para "confort" o "para dormir". En un estudio, los recién nacidos cuyas madres fueron más sensibles a sus señales de hambre y saciedad ganaron significativamente menos peso entre los 6 y 12 meses de edad, que los de madres menos sensibles a estas señales. La enseñanza a los padres sobre métodos alternativos para calmar a un lactante, en lugar de la alimentación inmediata, puede ayudar en la prevención de la ganancia de peso rápida en el primer año de vida ${ }^{(31)}$.

Aproximadamente a los cinco y medio meses de edad, muchos bebés empiezan a alcanzar los cubiertos o el alimento mismo. El permitir que el niño trate y se alimente a sí mismo, de acuerdo a su desarrollo, puede ayudar a promover la autorregulación de la ingesta. Los padres pueden determinar qué alimentos son ofrecidos, mientras que el niño puede determinar qué alimentos son consumidos y la cantidad. Este enfoque es consistente con el estilo de PAPP "con autoridad", que ha sido utilizado con éxito como estrategia para la promoción de una dieta saludable, y que ha sido asociada a menor sobrepeso y obesidad que otros estilos de PAPP "indulgente", sin mucho control, o "autoritario", con control excesivo ${ }^{(28)}$.

A medida que la alimentación del niño progresa, los padres pueden ayudar a niños en el proceso de autorregulación de la ingesta de energía ayudándoles con estímulos pertinentes como ofreciendo porciones adecuadas y estimulándolos a evaluar su saciedad, en lugar de alentándolos a comer "un bocado más". La Academia Americana de Pediatría sugiere que los niños mayores participen en la selección de alimentos dentro 
de las opciones de comida saludable proporcionada por los padres, y que sean ellos los que determinen cuánto se consume en cada comida ${ }^{(32)}$

La restricción excesiva de alimentos apetecibles también puede tener efectos deletéreos sobre el consumo de energía de los niños. Los niños mayores que experimentan una sobrerrestricción de ciertos alimentos o exceso de control parental durante las comidas pueden también tener dificultad en identificar sus propias señales de saciedad y en mantener su capacidad de autorregular la ingesta ${ }^{(33)}$. En preescolares, una vez que se elimina la restricción excesiva a golosinas o alimentos de refrigerio se puede observar un mayor consumo de estos. Además, los estudios sobre el peso de preescolares son mayores en niños cuyos padres informaron un mayor número de prácticas de restricción a estos alimentos de refrigerio. Prácticas menos restrictivas son más conducentes a una ingesta de calorías más equilibrada en niños de la misma edad. Por otro lado, un estilo de PAPP muy permisivo (indulgente y al margen) se ha asociado negativamente con la ingesta de una dieta saludable (más frutas y verduras) y un peso corporal más saludable ${ }^{(33,34)}$.

\section{CONCLUSIONES}

La epidemia de obesidad infantil continua avanzando, el problema es evidente cada vez en edades más tempranas, y el sobrepeso antes de los dos años de vida es ya predictivo del sobrepeso más adelante. Es también claro que muchos de los patrones de consumo alimentario asociados con la obesidad infantil empiezan muy tempranamente, y que estos a su vez están asociados con prácticas específicas de alimentación por parte de los padres.

Por un lado, la obesidad en la primera infancia es alarmante. Esto nos indica que nuestras estrategias deben enfocarse más en la prevención; y que nuestras intervenciones alimentarias y dietéticas deben empezar mucho más temprano de lo que hemos intentado hasta ahora. Por otro lado, nos debe servir de aliento el hecho de que las intervenciones antes de los dos años de vida tienen el potencial de ser tanto más efectivas como más eficaces.

La mayor parte de intervenciones para el manejo y prevención de la obesidad se han concentrado en adultos y niños mayores. En esta revisión señalamos los factores asociados con la obesidad infantil que son potencialmente modificables, y que requieren atención desde el nacimiento. Es importante notar que estos factores están asociados con la obesidad, y no necesariamente implican una relación causal. Por tanto, es importante emprender estudios prospectivos de intervención que tengan como metas actuar sobre estos factores modificables. Es mucho más fácil establecer patrones apropiados que el intentar cambiarlos, y no hay etapa más plástica en la vida para hacer esto que los primeros dos años.

Fuentes de financiamiento: autofinanciado.

Conflictos de interés: los autores declaran no tener conflictos de interés.

\section{REFERENCIAS BIBLIOGRÁFICAS}

1. The Organization for Economic Co-operation and Development (OECD). Obesity update 2012 [Internet]. Paris: OECD; 2012 [citado el 15 julio del 2012]. Disponible en: http://www.oecd.org/els/ healthpoliciesanddata/49716427.pdf

2. Moss BG, Yeaton WH. Young children's weight trajectories and associated risk factors: results from the Early Childhood Longitudinal StudyBirth Cohort. Am J Health Promot. 2011;25(3):190-8.

3. Winter JD, Langenberg P, Krugman SD. Newborn adiposity by body mass index predicts childhood overweight. Clin Pediatr (Phila). 2010;49(9):866-70.

4. Moss BG, Yeaton WH. U.S. children's preschool weight status trajectories: patterns from 9-month, 2-year, and 4-year Early Childhood Longitudinal Study-Birth cohort data. Am J Health Promot. 2012;26(3):172-5.

5. Stettler N, Iotova V. Early growth patterns and long-term obesity risk. Curr Opin Clin Nutr Metab Care. 2010;13(3):294-9.

6. Ogden CL. A review of prevalence and trends in childhood obesity in the United States. In: O'Dea JA, Eriksen M, eds. Childhood Obesity Prevention: International Research, Controversies, and Interventions. New York: Oxford University Press; 2010. p. 84-94.

7. Pajuelo-Ramirez J, Miranda-Cuadros M, Campos-Sanchez M, Sanchez-Abanto J. Prevalencia de sobrepeso y obesidad en niños menores de cinco años en el Perú 2007-2010. Rev Peru Med Exp Salud Publica. 2011;28(2):222-7.

8. Leao LS, Araujo LM, de Morales LT. Prevalência de obesidade em escolares de Salvador, Bahia. Arq Bras Endocrinol Metab. 2003;47(2):151-7.

9. Saldiva SR, Escuder MM, Venancio SI, Benicio MH. Prevalence of obesity in preschool children from five towns in Sao Paulo State, Brazil. Cad Saude Publica. 2004;20(6):1627-32.

10. Simon VG, Souza JM, Souza SB. Breastfeeding, complementary feeding, overweight and obesity in preschool children. Rev Saude Publica. 2009;43(1):60-9.

11. Conde WL, Monteiro CA. Secularchanges in overweight and obesity among Brazilian adolescents from 1974/75 to 
2002/03. In: O’Dea JA, Eriksen M, eds. Childhood Obesity Prevention: International Research, Controversies, and Interventions. New York: Oxford University Press; 2010. p. 144-51.

12. Ogden CL, Carroll MD, Curtin LR, Lamb MM, Flegal KM. Prevalence of high body mass index in US children and adolescents, 2007-2008. JAMA. 2010;303(3):242-9.

13. Dattilo AM, Birch L, Krebs NF, Lake A, Taveras EM, Saavedra JM. Need for early interventions in the prevention of pediatric overweight: a review and upcoming directions. J Obes. 2012;2012:123023.

14. Durmuş B, van Rossem L, Duijts L, Arends LR, Raat H, Moll HA, et al. Breast-feeding and growth in children until the age of 3 years: the Generation $R$ Study. Br J Nutr. 2011;105(11):1704-11.

15. Black RE, Allen LH, Bhutta ZA, Caulfield LE, de Onis M, Ezzati M, et al. Maternal and child undernutrition: global and regional exposures and health consequences. Lancet. 2008;371(9608):243-60.

16. Koletzko B, von Kries R, Closa R, Escribano J, Scaglioni S, Giovannini M, et al. Can infant feeding choices modulate later obesity risk? Am J Clin Nutr. 2009;89(5):1502S-8S.

17. Li R, Magadia J, Fein SB, GrummerStrawn LM. Risk of bottle-feeding for rapid weight gain during the first year of life. Arch Pediatr Adolesc Med. 2012;166(5):431-6.

18. Grummer-Strawn LM, Scanlon KS, Fein SB. Infant feeding and feeding transitions during the first year of life. Pediatrics. 2008;122 Suppl 2:S36-S42.

19. Beauchamp GK, Mennella JA. Flavor perception in human infants: development and functional significance. Digestion. 2011;83 Suppl 1:1-6.
20. Briefel RR, Kalb LM, Condon E, Deming DM, Clusen NA, Fox MK, et al. The Feeding Infants and Toddlers Study 2008: study design and methods. J Am Diet Assoc. 2010;110(12 Suppl):S16-26.

21. Dubois L, Farmer AP, Girard M, Peterson K. Preschool children's eating behaviours are related to dietary adequacy and body weight. Eur J Clin Nutr. 2007;61(7):846-55.

22. Siega-Riz AM, Deming DM, Reidy KC, Fox MK, Condon E, Briefel RR. Food consumption patterns of infants and toddlers: where are we now? J Am Diet Assoc. 2010;110(12 Suppl):S38-51.

23. Deming DM, Reidy KC, Briefel RR, Fox MK, Condon E. The Feeding Infants and Toddlers Study (FITS) 2008: Dramatic changes in the amount and quality of vegetables in the diet occur after the first year of life [abstract]. Conference Proceedings \& Specific Issues 2012: Research abstracts from Experimental Biology 2012. San Diego, CA: Nestlé Nutrition Institute. 2012.

24. Ventura AK, Mennella JA. Innate and learned preferences for sweet taste during childhood. Curr Opin Clin Nutr Metab Care. 2011;14(4):379-84.

25. Mennella JA, Ventura AK. Early feeding: setting the stage for healthy eating habits. Nestle Nutr Workshop Ser Pediatr Program. 2011;68:153-63.

26. Schaal B, Marlier L, Soussignan R. Human foetuses learn odours from their pregnant mother's diet. Chem Senses. 2000;25(6):729-37.

27. Birch LL, Anzman-Frasca S. Learning to prefer the familiar in obesogenic environments. Nestle Nutr Workshop Ser Pediatr Program. 2011;68:187-96.

28. Sleddens EF, Gerards SM, Thijs C, de Vries NK, Kremers SP. General parenting, childhood overweight and obe- sity-inducing behaviors: a review. Int J Pediatr Obes. 2011;6(2-2):e12-e27.

29. Dollberg S, Lahav S, Mimouni FB. A comparison of intakes of breastfed and bottle-fed infants during the first two days of life. J Am Coll Nutr. 2001;20(3):209-11.

30. Brotanek JM, Schroer D, Valentyn L, Tomany-Korman S, Flores G. Reasons for prolonged bottle-feeding and iron deficiency among Mexican-American toddlers: an ethnographic study. Acad Pediatr. 2009;9(1):17-25.

31. Worobey J, Lopez MI, Hoffman DJ. Maternal behavior and infant weight gain in the first year. J Nutr Educ Behav. 2009;41(3):169-75.

32. Kleinman RE. Pediatric nutrition handbook. 6th ed. Elk Grove Village, IL: American Academy of Pediatrics; 2009.

33. Frankel LA, Hughes SO, O'Connor TM, Power TG, Fisher JO, Hazen NL. Parental Influences on Children's Self-Regulation of Energy Intake: Insights from Developmental Literature on Emotion Regulation. J Obes. 2012;2012:327259.

34. Hoerr SL, Hughes SO, Fisher JO, Nicklas TA, Liu Y, Shewchuk RM. Associations among parental feeding styles and children's food intake in families with limited incomes. Int J Behav Nutr Phys Act. 2009;6:55.

Correspondencia: José M. Saavedra

Dirección: 12 Vreeland Rd. Florham Park, NJ 07932-0697, EEUU.

Correo electrónico:jose.saavedra@us.nestle.com

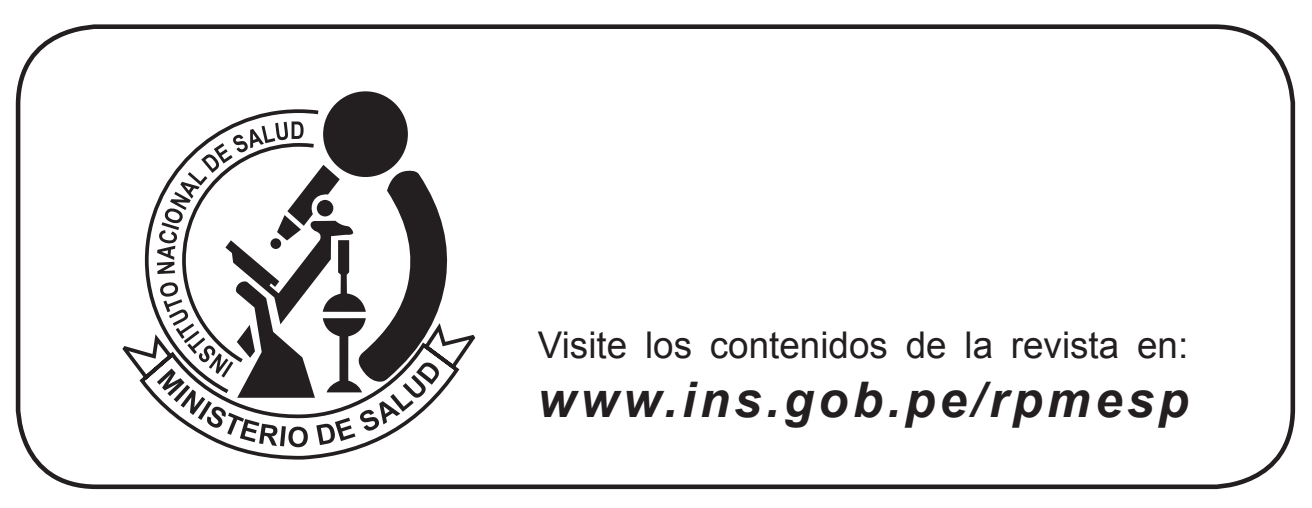

